\title{
Intérêt des traitements pharmacologiques symptomatiques des maladies neuromusculaires de l'enfont
}

\author{
Jean-Marie Cuisset, Ammélie Hamain, Alexandra Binoche, Stéphanie Coopman, Louis Vallée, \\ Sylvie N'Guyen
}

Les traitements pharmacologiques symptomatiques représentent un appoint utile dans la prise en charge des maladies neuromusculaires, qu'ils soient ciblés sur un processus physiopathologique corolaire de la maladie (fibrose musculaire, oxydation), sur un symptôme physique tel que la myotonie, la douleur, ou sur les troubles cognitifs liés à l'atteinte centrale associée (dystrophinopathies, dystrophie myotonique de Steinert). Ils permettent d'améliorer l'espérance et la qualité de vie, en attendant les progrès des thérapies curatives émergentes.

Jean-Marie Cuisset Louis Vallée Sylvie N'Guyen

Service de Neurologie Pédiatrique, hôpital Roger-Salengro, CHRU, Lille, France

Amélie Hamain

Centre de Rééducation

Pédiatrique Marc Sautelet,

Villeneuve d'Ascq, France

Alexandra Binoche

Service de réanimation

Pédiatrique, hôpital

Jeanne de Flandre,

CHRU, Lille, France

Stéphanie Coopman

Antenne Pédiatrique

du Centre d'Investigations

Cliniques, hôpital Jeanne

de Flandres, CHRU, Lille,

France

jean-marie.cuisset@

chru-lille.fr
La prise en charge thérapeutique des maladies neuromusculaires (MNM) de l'enfant est essentiellement symptomatique et multidisciplinaire, associant les aspects orthopédique, respiratoire, nutritionnel, digestif, cardiaque et la gestion de la douleur. Dans l'attente de la pleine efficience des thérapies curatives innovantes, susceptibles d'aboutir à une médecine moléculaire ciblée sur le gène, voire une médecine personnalisée spécifique de la mutation génique ou d'autres caractéristiques liées au patient (terrain génétique, pharmacocinétique, physiopathologie...), il parait donc bénéfique de prescrire, de façon optimisée, pour une maladie neuromusculaire donnée, tous les traitements pharmacologiques symptomatiques possibles. Une telle démarche appliquée dès la fin des années 1980 aux patients atteints de dystrophie musculaire de Duchenne (DMD), a permis de doubler leur espérance de vie en 20 ans. Les suppléments vitaminiques ou nutritionnels, les traitements symptomatiques concernant une complication ciblée sur un seul appareil (par exemple les troubles du transit intestinal) ne seront pas traités ici.

\section{Traitements symptomatiques ciblant le processus dystrophique}

\section{Fibrose musculaire}

Les corticoïdes représentent le premier traitement prouvé efficace dans la DMD, en retardant l'âge de perte de la marche [1]. Les mécanismes évoqués sont la stimulation de la prolifération des myoblastes, une stabilisation des protéases liées aux lysosomes et au sarcolemme, et surtout une diminution de la fibrose par effet immunosuppresseur et anti-inflammatoire [2]. Il existe deux protocoles principaux : l'un utilisant le deflazacort (Calcort $\left.{ }^{\circledast}\right)$ à une posologie de $0,9 \mathrm{mg} / \mathrm{kg} /$ jour $(0,8 \mathrm{mg} / \mathrm{kg}$ à partir de 10 ans, $0,55 \mathrm{mg} / \mathrm{kg}$ à partir de 15 ans et $0,5 \mathrm{mg} / \mathrm{kg}$ au-delà de 18 ans) [3] ; l'autre utilisant la prednisone $\left(\right.$ Cortancyl $\left.{ }^{\circledR}\right): 0,75 \mathrm{mg} / \mathrm{kg} /$ jour pendant 6 mois à 1 an, puis $0,75 \mathrm{mg} / \mathrm{kg}$ un jour sur 2 pendant 1 à 2 ans [4]. Les effets primaires de la corticothérapie sont multiples : recul de l'âge de perte de la marche de 12 à 14,5 ans [5]; amélioration de la fonction ventilatoire [6] ; âge d'apparition de la cardiomyopathie différé (15,2 ans versus 13,1 ans) [7] ; diminution du risque de scoliose (20\% vs $92 \%$ ) [8] ; réduction du taux de mortalité [9]. Les effets secondaires observés sont ceux d'une corticothérapie classique: fractures osseuses et tassements vertébraux, surpoids, retard statural, cataracte (infraclinique), troubles du comportement, hyperphagie, syndrome cushingoïde, hirsutisme, troubles gastriques [3]. Néanmoins, la variabilité de réponse des patients DMD à la corticothérapie, et le fait que $42 \%$ d'entre eux âgés de plus de 10 ans doivent interrompre les corticoïdes en raison d'effets secondaires a incité à rechercher d'autres traitements pharmacologiques symptomatiques.

\section{Oxydation}

L'idébénone, analogue synthétique du coenzyme $\mathrm{Q}_{10}$, puissant anti-oxydant et inhibiteur de la peroxydation lipidique susceptible de stimuler le flux d'électron mitochondrial et la production d'énergie a démontré une efficacité symptomatique sur des paramètres ventilatoires dans un essai de phase III chez des patients DMD d'un âge compris entre 10 et 18 ans et ne recevant pas de corticoïdes : 
stabilisation du débit expiratoire de pointe, diminution de l'altération de la capacité vitale forcée et du volume expiratoire forcé [10]. Une analyse a posteriori récente de cet essai a démontré une fréquence moindre d'infections bronchopulmonaires chez les patients traités par idébénone en comparaison du groupe placebo [11].

\section{Traitements ciblés sur un symptôme physique}

\section{Myotonie}

Qu'il s'agisse d'une dystrophie myotonique de Steinert ou d'une myotonie non dystrophique (paramyotonie congénitale et myotonie aggravée par le potassium liées au gène SCN4A ; myotonies congénitales $\mathrm{de}$ Thomsen et de Becker liées au gène CLCN1), la myotonie est une cible thérapeutique d'intérêt (Figure 1). La myotonie, quelle qu'en soit l'étiologie, résulte in fine d'une activation phasique des canaux $\mathrm{Na}^{+}$qui induit des trains de potentiel d'action haute fréquence au niveau de la fibre musculaire, corollaire électrophysiologique de la myotonie. La mexilétine, anti-arythmique Ib de la classification de VaughanWilliams, en bloquant les canaux $\mathrm{Na}^{+}$, diminue la durée du potentiel d'action et n'a pas (ou peu) d'action sur l'intervalle QT, et constitue donc un anti-myotonique majeur. Il se prescrit à une posologie de $15 \mathrm{mg} / \mathrm{kg} /$ jour chez le nourrisson, $8 \mathrm{mg} /$ $\mathrm{kg} /$ jour chez le grand enfant, en trois prises (200 mg, trois fois par jour chez l'adulte) [19]. Sa prescription impose un ECG à $\mathrm{H}_{0}, \mathrm{H}_{24}$, et $\mathrm{H}_{48}$ pour évaluation de l'espace PR et du segment QT corrigé.

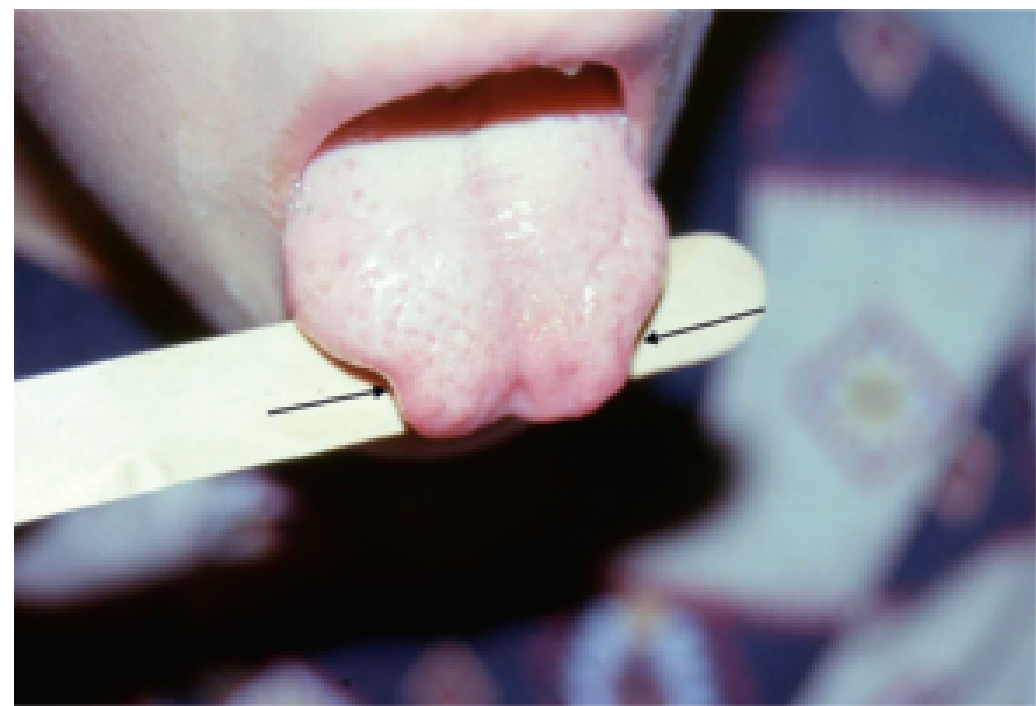

Figure 1

Anneau de constriction myotonique au niveau lingual (flèches) chez un patient Steinert. Les accès myotoniques de la langue chez ce patient étaient à l'origine de troubles de la déglutition, qui ont totalement régressé après l'introduction de la mexilétine.
Ses principaux effets secondaires sont: vertiges, dysgueusie, vomissements, somnolence et ataxie. La mexilétine a été prouvée efficace dans la myotonie congénitale [12], et dans la myotonie des patients adultes atteints de dystrophie myotonique de Steinert [13].

\section{Ostéoporose}

Les biphosphonates intraveineux peuvent être bénéfiques dans les maladies neuromusculaires prédisposant à l'ostéoporose d'immobilisation, en particulier chez les patients DMD ou ceux présentant une amyotrophie spinale de type 2 . Les deux indications principales sont les fractures pathologiques et le syndrome algique chronique (d'expression essentiellement nocturne). Il convient, 10 à 15 jours avant la cure, d'éliminer une hypocalcémie latente en prescrivant calcémie, protidémie et étude du rapport calciurie/créatininurie (normale $>0,2$ ). Il est possible d'utiliser soit le pamidronate (Arédia ${ }^{\circledR}$ ) : $0,5 \mathrm{mg} / \mathrm{kg}$ deux fois (en deux jours consécutifs), à perfuser dans $500 \mathrm{ml}$ de glucosé à $5 \%$ sur quatre heures; soit le zonidronate $\left(\right.$ Zométa $^{\circledR}$ ) : $0,5 \mathrm{mg} / 10 \mathrm{~kg}$ (maximum : $4 \mathrm{mg}$ ) en injection IV unique en 15 minutes dans $100 \mathrm{ml}$ de glucosé à $5 \%$. Les cures d'Arédia se renouvèlent tous les quatre mois, celles de Zométa tous les six mois. Les principaux effets secondaires sont : syndrome grippal, nausées, fatigue, hypocalcémie, hypozincémie (calcémie et zincémie sont à contrôler systématiquement 48-72 heures après la cure). Gordon et al. ont établi sur une série de 44 patients DMD bénéficiant d'une corticothérapie, que ceux qui recevaient des cures de biphosphonates IV avaient une durée de vie augmentée. Ils suggèrent que les biphosphonates ralentiraient le processus dystrophique [14].

\section{Douleur}

En ce qui concerne le syndrome algique, Wagner et al. ont objectivé pour les biphosphonates un taux d'efficacité de $98 \%$ (dans $80 \%$ des cas disparition de la douleur, durable dans $20 \%$ des cas, transitoire (pendant 1 à 3 mois) dans $60 \%$ des cas) [15].

\section{Rétablissement d'une transmission neuromusculaire efficiente}

Les syndromes myasthéniques congénitaux (SMC) [16] regroupent un ensemble hétérogène d'affections génétiquement déterminées responsables d'un dysfonctionnement de la transmission neuromusculaire à l'origine d'une faiblesse musculaire accentuée par l'effort. Un début précoce (presque toujours avant deux ans) et une atteinte oculo-bulbaire (ptosis et/ou ophtalmoplégie; dysphonie, troubles de la déglutition, parésie faciale) sont des éléments 
sémiologiques très évocateurs. Plus de 20 gènes à l'origine des SMC ont été identifiés. Leurs mutations peuvent être à l'origine d'une anomalie du récepteur musculaire nicotinique à l'acétylcholine (RAch), d'un défaut de protéines présynaptiques, d'anomalies de protéines associées à la lame basale présynaptique, de troubles du développement ou de la stabilité de la plaque motrice, ou d'anomalies de protéines intervenant dans la glycosylation. Différents traitements symptomatiques visant au rétablissement d'une transmission neuromusculaire efficiente sont disponibles : anticholinestérasiques, 3-4 diaminopyridine (DAP) de mode d'action présynaptique (inhibition des canaux potassiques de la terminaison nerveuse, libération accrue de vésicules d'acétylcholine), molécules réduisant le temps d'ouverture du RAch (quinidine, fluoxétine), $\beta 2$ mimétiques (éphédrine, salbutamol). Leur choix dépend du gène en cause. Les anticholinestérasiques sont efficaces dans les SMC liés aux gènes codant pour une sous-unité du RAch, aux gènes RAPSN (rapsyne), GFPT1 et CHAT. Ils sont contre-indiqués dans les déficits en acétylcholine estérase ( $\mathrm{AChE}$; liés au gène COLQ) et les syndromes du canal lent souvent décelés par une réponse répétitive après stimulation unique à l'électroneuromyogramme, et le déficit en DOK7. La 3-4 DAP est efficace dans les SMC post- synaptiques : déficit en RAch à cinétique normale, déficit en rapsyne, déficit en MuSK. Elle est un complément utile des anticholinestérasiques. Les $\beta 2$ mimétiques sont utiles dans les déficits en DOK7 et en COLQ. La quinidine et la fluoxétine sont efficaces dans les syndromes du canal lent (liés à et $C H R N E$ ).

\section{Atteinte cognitive}

L'atteinte cognitive de certaines maladies neuromusculaires peut être une cible thérapeutique bénéfique. Dans les dystrophinopathies, les troubles cognitifs constants et précoces sont dominés par les difficultés attentionnelles. Le trouble hyperactif avec déficit attentionnel (THADA) existerait chez un tiers des patients DMD, le déficit attentionnel isolé chez $15 \%$ d'entre eux [17], pouvant justifier la prescription du méthylphénidate (Ritaline ${ }^{\circledR}$ ou équivalents). Dans la dystrophie myotonique de Steinert, les troubles comportementaux et cognitifs sont également fréquents : phobies, troubles de l'humeur, troubles anxieux et THADA dans près d'un tiers des cas. L'intérêt du méthylphénidate chez ces patients Steinert avec THADA a également été rapporté [18, 19].

\section{Conclusion}

L'espoir majeur suscité par la perspective des thérapies à visée curative depuis la fin des années 1980, demeure à ce jour contrarié, par des problèmes pratiques ou méthodologiques. Ainsi, dans la dystrophie musculaire de Duchenne (DMD), les essais thérapeutiques relevant du saut d'exons ou de la translecture d'un codon stop se heurtent à des âges d'inclusion hétérogènes, à des co-médications imposées (corticoïdes), et à des critères d'efficacité insuffisamment pertinents (performances motrices plutôt que quantité de dystrophine produite de novo) [20]. De même dans l'amyotrophie spinale typique (SMA), les essais procédant d'une correction d'épissage du gène SMN2 par oligonucléotides anti-sens sont confrontés à une fenêtre temporelle d'efficacité thérapeutique étroite et à la nécessité d'une diffusion systémique en raison d'une atteinte multiorgane [21]. Ces restrictions justifient, en attendant une pleine efficacité des traitements curatifs, le recours à des traitements symptomatiques optimisés.

Interest of symptomatic pharmacological treatments in childhood neuromuscular disorders

\section{LIENS D'INTÉRÊT}

Les auteurs déclarent n'avoir aucun lien d'intérêt concernant les données publiées dans cet article.

\section{RÉFÉRENCES}

1. Dubowitz V. $75^{\text {th }}$ European neuromuscular centre international workshop on the treatment of muscular dystrophy, 10-12 December 1999, Naarden, The Netherlands. Neuromuscul Disord 2000 ; 10 : 313-20.

2. Zatz M, Pavanello Rde C, Vainzof Met al. Steroids in Duchenne muscular dystrophy. Neuromuscul Disord 1992; 2 : 59.

3. Manzur AY, Kuntzer T, Pike M, et al. Glucocorticoid corticosteroids for Duchenne muscular dystrophy. Cochrane Database Syst Rev 2016 ; 5 : CD003725.

4. McAdam LC, Mayo AL, Alman BA, Biggar WD. The Canadian experience with long-term deflazacort treatment in Duchenne muscular dystrophy. Acta Myol 2012 ; 31 : 16-20.

5. Ricotti V, Ridout DA, Scott E, et al. Long-term benefits and adverse effects of intermittent versus daily glucocorticoids in boys with Duchenne muscular dystrophy. J Neurol Neurosurg Psychiatr 2013; $84: 698-705$.

6. Buyse GM, Goemans N, van den Hauwe M, et al. Effects of glucocorticoids and idebenone on respiratory function in patients with Duchenne muscular dystrophy. Pediatr Pulmonol 2013; 48 : 912-20

7. Barber BJ, Andrews JG, Lu Z, et al. Oral corticosteroids at onset of cardiomyopathy in Duchenne muscular dystrophy. $J$ Pediatr 2013; 163 : 1080-4e1.

8. Lebel DE, Corston JA, McAdam LC, et al. Glucocorticoid treatment for the prevention of scoliosis in children with Duchenne muscular dystrophy: long term follow up. J Bone Joint Surg Am 2013 ; 95 : 1057-61.

9. Schram G, Fournier A, Leduc H, et al. All-cause mortality and cardiovascular outcomes with prophylactic steroid therapy in Duchenne muscular dystrophy. J Am Coll Cardiol 2013; 61 : 948-54.

10. Buyse GM, Voit T, Schara U, et al. Efficacy of idebenone on respiratory function in patients with Duchenne muscular dystrophy not using glucocorticoids (DELOS): a double-blind randomised placebo-controlled phase 3 trial. Lancet 2015 ; 385 : 1748-56.

11. McDonald CM, Meier T, Voit T, et al. Idebenone reduces respiratory complications in patients with Duchenne muscular dystrophy. Neuromuscul Disord 2016 ; 26 : 473-80. 
12. Leheup B, Himon F, Morali A, et al. Intérêt de la mexilétine dans le traitement de la myotonie de Thomsen-Becker. Arch Fr Pediatr 1986 ; 43 : 49-50.

13. Logigian EI, Martens WB, Moxley IV RT, et al. Mexiletine is an effective antimyotonia treatment in myotonic dystrophy type 1 . Neurology 2010 ; 74 : 1441-8.

14. Gordon KE, Dooley JM, Sheppard KM, et al. Impact of biphosphonates on survival for patients with Duchenne muscular dystrophy. Pediatrics 2011; 127 : e353-8.

15. Wagner S, Poirot I, Vuillerot C, Berard C. Tolerance and effectiveness on pain control of Pamidronate ${ }^{\circledR}$ intravenous infusions in children with neuromuscular disorders. Ann Phys Rehab Med 2011 ; 54 : 348-58.

16. Eymard B, Stojkovic T, Sternberg D, et al. Syndromes myasthéniques congénitaux : difficultés diagnostiques, évolution et pronostic, thérapeutique. L'expérience du réseau national Syndromes myasthéniques congénitaux. Rev Neurol 2013 ; 169 : S45-5.
17. Pane M, Lombardo ME, Alfieri P, D'Amico A, et al. Attention deficit hyperactivity disorder and cognitive function in Duchenne muscular dystrophy: phenotype-genotype correlation. J Pediatr $2012 ; 161$ : 705-9

18. Douniol M, Jacquette A, Cohen D, et al. Psychiatric and cognitive phenotype of childhood myotonic dystrophy type 1. Dev Med Child Neurol 2012 ; 54 : 905-11.

19. Ishigaki K, Muto A, Osawa M. Clinical features and care of patients with congenital and childhood onset myotonic dystrophy. Rinsho Shinkeigaku 2012; 52 : 1264-6.

20. Merlini L, Sabatelli P. Improving clinical trial design for Duchenne muscular dystrophy. BMC Neurol 2015; 15 : 153

21. Wirth B, Barkats M, Martinat C, et al. Moving towards treatments for spinal muscular atrophy: hopes and limits. Expert Opin Emerging Drugs 2015 ; 20 : 352-6.

\section{www.myobase.org}

Ce portail documentaire rassemble les documents sur les maladies neuromusculaires, les situations de handicap qu'elles génèrent et leurs aspects psychologiques.

Une sélection

pertinente de plus

de $\mathbf{5 5 0 0 0}$ notices

bibliographiques

$>$ articles de la littérature

biomédicale

et psycho-sociale

$>$ livres, thèses...

$>$ guides d'associations

et rapports

institutionnels

d'agences internationales

$>$ brèves en français,

synthèses des articles médico-scientifiques internationaux les plus pertinents

$>$ publications AFM. Téléthon destinées aux professionnels de santé ou aux personnes atteintes de maladie neuromusculaire et à leur entourage

\section{UN OUTIL \\ ERGONOMIQUE, UNE INTERFACE BILINGUE}

- Laissez-wous guider par les tutoriels

- Lancez une recherche et affinez votre sélection grâce aux filtres

\begin{tabular}{|c|c|}
\hline \multicolumn{2}{|c|}{ TOUT MYOBASE } \\
\hline Anotherd & $\mathrm{Ol}$ \\
\hline \multicolumn{2}{|c|}{ Aectherctie evencite } \\
\hline \multirow[t]{3}{*}{ Minto } & FILTRES \\
\hline & Type de dooventert \\
\hline & 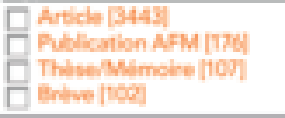 \\
\hline
\end{tabular}

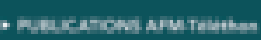

\section{s eatuss}

+ cocournts ce sramitst

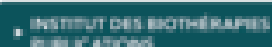
"hancitions

- Téléchargez le texte intégral des documents libres de droit

- Partagez les résultats de votre recherche

\section{DIFFÉRENTS ACCÉS AUX NOUVEAUTES QUOTIDIENNES}
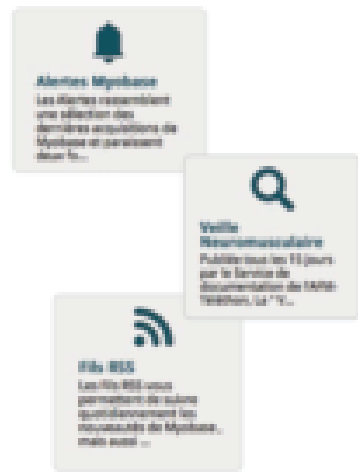

- Cliquez sur I'onglet thématique qui vous convient (haut de la page d'accueil)

- Abonnez-wous aux alertes thématiques. Créez vos alertes personnalisées en ouvrant un compte personnel

- Téléchargez la Veille Neuromusculaire

- Abonnez-vous aux flux RSS 